\title{
MAGNETIC X-RAY SCATTERING
}

\author{
C. VetTier
}

European Synchrotron Radiation Facility, BP 220, 38043-Grenoble Cedex, France

It was proposed in the 1950's that X-rays could be used to probe magnetization densities. After some experimental demonstrations of the technique, applications have shown that the use of magnetic X-ray scattering combined with synchrotron radiation can lead to important new discoveries in the magnetism of solid materials. Several important features have emerged, especially the resonant magnetic X-ray scattering: large enhancements of scattered intensities, site and species selectivity, X-ray energy and polarization dependence. These properties make it possible to study new phenomena: details of magnetic structures of materials including micro-crystals, new aspects of magnetic phase transitions and surface magnetism.

PACS numbers: $61.10 . \mathrm{Dp}, 75.25 .+\mathrm{z}, 75.40 .-\mathrm{s}$

\section{Introduction}

The use of photons as a probe for magnetization densities dates as back as the last century when the magneto-optics was established $[1,2]$. These phenomena correspond to the rotation of the polarization of light upon reflection or transmission through a magnetized medium. With hard X-rays, such effects are difficult to observe because the index of refraction for X-rays is very close to unity. It is only near the absorption, edges of materials that the index of refraction differs from unity: this leads to magnetic X-ray absorption and magnetic dichroism effects $[3,4]$. Since the absorption cross-section and the scattering amplitudes are related through the optical theorem [5], magnetic effects are to be observed also in $X$-ray scaltering. Indeed, the first evidence for magnetic scattering effects was given in 1972 by de Bergevin and Brunel [6] who could observe a weak magnetic Bragg peak from a single crystal of $\mathrm{NiO}$ with a standard X-ray tube. Later on, synchrotron radiation became available, and although stronger scattered intensities could be observed, magnetic $r$-ray scattering was still regarded as a curiosity, until resonant magnetic scattering was discovered $[7,8]$. The large enhancements of magnetic intensity (up to 6 or 7 orders of magnitude) that can be observed at certain absorption edges make it possible to envisage new experiments that could not be done before even with neutrons.

The purpose of this lecture is to present a survey of the main features and ranges of applications of magnetic X-ray scattering. The paper is organized as 
follows: in the next section, the basic ingredients of the magnetic scattering are presented while putting the emphasis on the physics of the process. In the third and fourth sections, we will discuss the information that can be obtained from nonresonant and resonant scattering magnetic techniques by reviewing some experimental results. To emphasize the relevance of magnetic $\mathrm{X}$-ray scattering to the magnetic propertics of solids, we will restrict ourselves to the Bragg scattering. Finally, some perspectives for the future use of magnetic X-ray scattering will be outlined.

\section{Magnetic X-ray scattering cross-sections}

In a purely nonrelativistic limit, X-rays couple only to electronic charges in solids and no information can be obtained on the spin densities. Ilowever, the complete relativistic treatment of the interaction of photons with charged particles depends on the spin of these particles [9]. Because the space and spin components cannot be separated in the relativistic theory, a perturbation of the electron movement has an effect which depends on its spin state. The amplitude of these effects during the scattering process can be parametrized by a quantity that reflects the relativistic character of the collision: the clange in the wave vector before and after the collision, normalized to the Compton wave vector: $\hbar K / m c$, where $K$ is the scattering vector $K=k_{\mathrm{i}}-k_{\mathrm{i}}$. Obviously, such correction vanishes in the forward direction where X-ray absorption or reflection experiments are made. The magnetic effects (magnetic Kerr effect, magnetic dichroism) observed in the forward direction must be related to resonant effects which take into account the characteristic energies of the electrons when they are bound in atoms. In a simple picture, when photoelectric absorption takes place, an electron is promoted into an empty shell, the symmetry of which depends on the orientation of the local magnetization. This opens new channcls for the scattering process and provides new contributions to the $\mathrm{X}$-ray scaltering amplitude. Therefore, two regimes for the interaction of X-rays with magnetization are distinguished depending on the photon energy: a high energy regime, where the characteristic atomic cnergies are neglected leading to the normal magnetic $X$-ray scattering, and a resonant regime where the photon energy is tuned close to these characteristic energies, where both the Bragg scattering experiments and absorption measurements are sensitive to magnetization densities.

\subsection{Magnelic $X$-ray scallering}

A series of articles [9-13] have described the approximations and the formalism involved in deriving expressions for the scattering amplitudes. IIere, we will limit ourselves to a description of the processes. The interaction between photons and spin and orbital momentum densities arise from relativistic interactions. Platzman and Tzoar [11] were the first to predict that X-rays could be used to determine magnetic structures. Simple classical pictures by de Bergevin and Brunel [9] indicate how the clectronic spins interact with the electromagnctic fields associated with the photon radiation: the electron magnetic moment is accelerated 
and reradiates electromagnetic components of the scattered wave. If the photon energy is much larger than the characteristic atomic energies $[9,12]$ the scattering amplitude $f^{\text {mag }}$ takes the simple form

$$
f^{\mathrm{mag}}=-\mathrm{i} r_{0} \frac{\hbar \omega}{m c^{2}}\left(\frac{1}{2} A \cdot L_{j}(K)+B \cdot S_{j}(K)\right),
$$

where $\boldsymbol{L}_{j}(K)$ and $S_{j}(K)$ represent the Fourier transforms of the orbital and spin momentum densities, respectively and $K$ is the momentum transfer. $r_{0}$ is the classical electron radius; $L$ and $S$ are in units of $\hbar$.

The quantities $\boldsymbol{A}$ and $\boldsymbol{B}$ are well-defined complex vectors which contain the wave vectors and polarization states of the incident and scattered photons. It is therefore possible to normalize scattered intensities and to extract magnetic moment in units of $\mu_{\mathrm{B}} . \boldsymbol{A}$ and $\boldsymbol{B}$ depend on the scattering geometry and can be varied accordingly. Because these two vectors are formally not identical, the spin momentum $\mu_{S}$ and the orbital momentum $\mu_{L}$ contribute differently to $f^{\text {mag }}$ and can be distinguished expcrimentally. Furthermore, the magnetic scattering amplitude is not diagonal in the two polarization states and mixes the different polarizations [13], contrary to the usual Thomson scattering, which is a convenient way to separate the two. It should be borne in mind that polarization analysis of scattered intensities yields also information about the direction of the Fourier components of $L(K)$ and $S(K)$. The magnetic amplitude in (1) can be expressed with a matrix whose basis vectors are the polarization vectors perpendicular and parallel to the scattering plane

$$
-\mathrm{i} \frac{\hbar \omega}{m c^{2}}\left(\begin{array}{cc}
A_{\sigma \sigma} & A_{\sigma \pi} \\
A_{\pi \sigma} & A_{\pi \pi}
\end{array}\right)
$$

where

$$
\begin{aligned}
& A_{\sigma \sigma}=S(K) \cdot\left(\widehat{k}_{\mathrm{f}} \times \widehat{k}_{\mathrm{i}}\right), \\
& A_{\sigma \pi}=-\frac{K^{2}}{2 k^{2}}\left[\left(\frac{L(K)}{2}+S(K)\right) \cdot \widehat{k}_{\mathrm{f}}+\frac{L(K)}{2} \cdot \widehat{k}_{\mathrm{i}}\right], \\
& A_{\pi \sigma}=\frac{K^{2}}{2 k^{2}}\left[\left(\frac{L(K)}{2}+S(K)\right) \cdot \widehat{k}_{\mathrm{i}}+\frac{L(K)}{2} \cdot \widehat{k}_{\mathrm{f}}\right], \\
& A_{\pi \pi}=\left[\frac{K^{2}}{2 k^{2}} L(K)+S(K)\right] \cdot\left(\widehat{k}_{\mathrm{f}} \times \widehat{k}_{\mathrm{i}}\right) .
\end{aligned}
$$

The total scattering cross-section contains the classical Thomson intensity, the purely magnetic contribution from (1) and an interference term between the magnetic amplitude and the Thomson scattering amplitude. The relative size of magnetic scattering amplitude to the charge amplitude is given by $\hbar K / m c$, which is of the order of a few $10^{-3}$ in usual diffraction experiments. Magnetic scattering is further reduced compared to the normal Thomson charge scattering because only the unpaired electrons take part in that process. As a result, in most cases the purely magnetic intensity is lower than the Thomson intensity by 7 or 8 orders of magnitude. Ilowever, in ferro- or ferrimagnetic materials, there always exists an interference term in the intensity between the charge scattering amplitude from the lattice and the magnetic scattering amplitude [15]. In such a case, it is possible 
to extract the magnetic amplitude by measuring $X$-ray intensities upon reversal of the magnetization. This technique, which is applicable even with unpolarized $\mathrm{X}$-rays, is equivalent to the polarized neutron scattering method for ferromagnets. Note that the magnetic amplitude in (1) is phase-slifted by $\pi / 2$ with respect to the charge scattering. In the case of realstructure factors (which imply both a centrosymmetric structure and a zero absorption), the interference is observed only in the case of circularly polarized X-ray beams. In the case of pure linear polarization $\sigma$, this asymmetry is given by

$$
R=\frac{2 \operatorname{Re}\left(F f^{\mathrm{mag}}\right)}{|F|^{2}}
$$

where $F$ is the classical crystal structure factor and $f^{\text {mag }}$ - the magnetic amplitude (1).

The scattering amplitude (1) gives also rise to magnetic inelastic X-ray scattering [16]: magnetic Compton scattering [16-18] or magnetic scattering by valence-electron excitations. The magnetic Compton scattering yields information on the distribution in $p$-space of unpaired electrons. The magnetic Compton signal can be extracted from the total scattering through the measurement of asymmetry ratio (3) with circularly polarized X-ray beams.

\subsection{Resonant magnetic X-ray scatlering}

The magnetic $\mathrm{X}$-ray scattering cross-section in (1) vanishes at zero momentum transfer and does not contribute to absorption effects, by direct application of the optical theorem. Therefore, the magnetization sensitive dichroic effects that take place near X-ray absorption edges correspond to resonant scattering processes, not described by the normal magnetic X-ray cross-section (1) but predicted by Blume [12]. Indeed, a resonant magnetization sensitive scattering was discovered by Gibbs and co-workers [7] and accounted for by IIannon and co-workers [19].

The appearance of magnetic dichroism and the enhancement of the magnetic scattering amplitude arise from the same mechanism: a promotion of an inner shell electron into available electronic states. Due to Pauli exclusion principle, the electric transitions are allowed only to unoccupied electronic states, which results in an exchange interaction sensitive to the magnetization of the final states. In this sense, the additional contribution to the cross-section arising from electric transitions is a probe of the local magnelic moments. By contrast with the nonresonant magnetic X-ray scattering, which originates from magnelic couplings, the resonant magnetic X-ray scattering and the dichroism can be analyzed in terms of electric multipole transitions between an atomic core level and either an unfilled atomic shell or a narrow electronic band.

The resonant magnetic scattering amplitude can be written in a simple and symmetric dependence on the magnetization direction and the polarization of the photon beams [19-21]. It contains all symmetric forms that can be formed with combinations of magnetization direction, the polarizations vectors and the wave vectors of the incident and final plotons [20]. The coefficients are given by the nature of the interaction, namely the promotion of an electron into an available 
electronic state above the Fermi level. If we restrict ourselves to the electric dipole transitions, the resonant part of the scattering amplitude can be written as [19]:

$$
\begin{aligned}
f_{\mathrm{E} 1}^{\text {res }} & =\frac{3 \lambda}{8 \pi}\left\{e_{\mathrm{f}}^{*} \cdot e_{\mathrm{i}}\left[F_{11}+F_{1-1}\right]-\mathrm{i}\left(e_{\mathrm{f}}^{*} \times e_{\mathrm{i}}\right) \cdot z_{j}\left[F_{11}+F_{1-1}\right]\right. \\
& \left.+\left(e_{\mathrm{f}}^{*} \cdot z_{j}\right)\left(e_{\mathrm{i}} \cdot z_{j}\right)\left[2 F_{10}-F_{11}-F_{1-1}\right]\right\} .
\end{aligned}
$$

$\boldsymbol{e}_{\mathrm{i}}$ and $\boldsymbol{e}_{\mathrm{f}}$ are the polarization vectors, implicitly contained in the vectors $\boldsymbol{A}$ and $\boldsymbol{B}$ for the nonresonant magnetic scattering in (1). The quantity $z_{j}$ represents the magnetization direction defined by the local moment on site $j$. The resonant amplitude (4) is governed by the $F_{l m}$. These coefficients contain matrix elements, which couple the ground state to the excited magnetic levels, and a resonant denominator. They are expressed as [19]

$$
F_{l m}=\sum_{a, b} \frac{p_{a} p_{a(b)} \Gamma_{x}(a M b ; \mathrm{EL})}{\left(E_{a}-E_{b}-\hbar \omega\right)-\mathrm{i} \Gamma / 2}
$$

where $a$ and $b$ are the initial and excited states of energy $E_{a}$ and $E_{b}$ respectively. $p_{a}$ gives the statistical probability for the initial state $a$, and $p_{a(b)}$ gives the probability that the excited state is vacant for transitions from $a . \Gamma$ is the total width for $b$, and $\Gamma_{x}(a M b ; \mathrm{EL})$ gives the partial width for EL decay from $b$ to $a$ when summed over $M$. It follows that the energy dependence of the amplitude (4) may reveal important details in the density of unoccupied states near the Fermi level. It is possible to extract exchange splitting and the induced polarization of conduction bands. The coefficients $F_{l m}$ can be calculated by modeling the density of states and wave functions, but there is no such a simple relationship between the scattered intensity and the magnetization magnitude as in the case of nonresonant magnetic $\mathrm{X}$-ray scattering (1) or neutron magnetic scattering.

The resonant amplitude $f_{\mathrm{E} 1}^{\text {mag }}(4)$ contains three different terms. The first one, independent of the quantization direction, corresponds to the classical anomalous scattering. The second one is linear in $z_{j}$ : it leads to magnetic scattering amplitude at the same place in reciprocal space as the usual magnetic scattering. Furthermore, it describes the magnetic X-ray circular dichroism [21] in absorption experiments $\left(k_{\mathrm{i}}=k_{\mathrm{f}}, e_{\mathrm{i}}=e_{\mathrm{f}}\right.$ ). The third term is quadratic in $z_{j}$. It will give second-harmonic magnetic Bragg peaks. It does not vanish with real $\boldsymbol{e}_{\mathrm{i}}$ and $\boldsymbol{e}_{\mathrm{f}}$ in the absorption case and leads to linear dichroism.

Although dipolar transitions are the strongest, the quadrupolar terms have to be considered, too. The corresponding scattering amplitude can be written as [20-24]:

$$
\begin{aligned}
f_{\mathrm{E} 2}^{\text {res }} & =\frac{5 \lambda}{8 \pi}\left\{\left(\widehat{k}_{\mathrm{f}} \cdot \widehat{k}_{\mathrm{i}}\right)\left(e_{\mathrm{f}}^{*} \cdot e_{\mathrm{i}}\right)\left(F_{22}+F_{2-2}\right)\right. \\
& -\mathrm{i}\left[\left(\widehat{k}_{\mathrm{f}} \cdot \widehat{k}_{\mathrm{i}}\right) \cdot\left(e_{\mathrm{f}}^{*} \times e_{\mathrm{i}}\right)+\left(\widehat{k}_{\mathrm{f}} \leftrightarrow e_{\mathrm{f}}^{*}, \widehat{k}_{\mathrm{i}} \leftrightarrow e_{\mathrm{i}}\right)\right]\left(F_{22}-F_{2-2}\right) \\
& -\left(\widehat{k}_{\mathrm{f}} \times \widehat{k}_{\mathrm{i}}\right) \cdot z_{\mathrm{i}}\left(e_{\mathrm{f}}^{*} \times e_{\mathrm{i}}\right) \cdot z_{j}\left(F_{22}+F_{2-2}\right) \\
& -\left[\left(\widehat{k}_{\mathrm{f}} \cdot \widehat{k}_{\mathrm{i}}\right)\left(e_{\mathrm{f}}^{*} \cdot z_{j}\right)\left(e_{\mathrm{i}} \cdot z_{j}\right)+\left(\widehat{k}_{\mathrm{f}} \leftrightarrow e_{\mathrm{f}}^{*}, \widehat{k}_{\mathrm{i}} \leftrightarrow e_{\mathrm{i}}\right)\right] \\
& \times\left[\left(F_{22}+F_{2-2}\right)-\left(F_{21}+F_{2-1}\right)\right]
\end{aligned}
$$




$$
\begin{aligned}
& +\mathrm{i}\left[\left(\widehat{k}_{\mathrm{f}} \cdot z_{j}\right)\left(\widehat{k}_{\mathrm{i}} \cdot z_{j}\right)\left(e_{\mathrm{f}}^{*} \times e_{\mathrm{i}}\right) \cdot z_{j}\right. \\
& \left.+\left(\widehat{k}_{\mathrm{f}} \leftrightarrow e_{\mathrm{f}}^{*}, \widehat{k}_{\mathrm{i}} \leftrightarrow e_{\mathrm{i}}\right)\right]\left[\left(F_{22}-F_{2-2}\right)-\left(F_{21}-F_{2-1}\right)\right] \\
& +\left[\left(e_{\mathrm{f}}^{*} \cdot \widehat{k}_{\mathrm{i}}\right)\left(k_{\mathrm{f}} \cdot z_{j}\right)\left(e_{\mathrm{i}} \cdot z_{j}\right)\right. \\
& \left.+\left(\widehat{k}_{\mathrm{f}} \cdot e_{\mathrm{i}}\right)\left(e_{\mathrm{f}}^{*} \cdot z_{j}\right)\left(\widehat{k}_{\mathrm{i}} \cdot z_{j}\right)\right]\left(F_{21}+F_{2-1}\right) \\
& -\mathrm{i}\left[\left(e_{\mathrm{f}}^{*} \cdot z_{j}\right)\left(\widehat{k}_{\mathrm{i}} \cdot z_{j}\right)\left(\widehat{k}_{\mathrm{f}} \times e_{\mathrm{i}}\right) \cdot z_{j}\right. \\
& \left.+\left(\widehat{k}_{\mathrm{f}} \cdot z_{j}\right)\left(e_{\mathrm{i}} \cdot z_{j}\right)\left(e_{\mathrm{f}}^{*} \times \widehat{k}_{i \mathrm{i}}\right) \cdot z_{j}\right]\left(F_{21}+F_{2-1}\right) \\
& +\left[\left(\widehat{k}_{\mathrm{f}} \cdot z_{j}\right)\left(\widehat{k}_{\mathrm{i}} \cdot z_{j}\right)\left(e_{\mathrm{f}}^{*} \cdot z_{j}\right)\left(e_{\mathrm{i}} \cdot z_{j}\right)\right] \\
& \left.\times\left[2 F_{20}-4\left(F_{21}+F_{2-1}\right)-\left(F_{22}+F_{2-2}\right)\right]\right\} .
\end{aligned}
$$

This scattering amplitude contains terms up to fourth order in $z_{j}$, which would lead to diffraction harmonics up to fourth order. In a scattering experiment, the existence of fourth-order magnetic Bragg peaks near a resonance is a signature of quadrupole transitions [19]. Scattering experiments allow an easy separation of the multipolar contributions to the resonance.

The resonant scattering amplitude does not depend explicitly on the orbital and the spin magnetizations. In (4) and (6), only the total magnetization direction $z_{j}$ appears. Ilowever, in the case of dipolar transitions, sum rules for the imaginary part of $f_{\mathrm{E} 1}^{\text {mag }}$ have been derived $[25,26]$. They indicate that the spin and orbital contributions to the magnetization can be obtained by integrating the $\operatorname{Im}\left(f_{\text {res }}^{\mathrm{mag}}\right)$ over two spin-orbit split edges, such as $L_{2}$ and $L_{3}$, or $M_{4}$ and $M_{5}$ edges.

At this point, it is worth discussing the amplitude of resonances and their applicability to Bragg scattering experiments. The magnitude of resonant effects is predicted to be the largest for the lowest order electric multipole transitions. The enhancement is even bigger if the final states correspond to the shell of unpaired electrons [19]. The resonant enhancement is further increased when the spin-orbit coupling splits the inner shell in a well-resolved doublet as the $L_{2}-L_{3}$ or $M_{4}-M_{5}$ edges. Therefore, resonant X-ray scattering is expected to be strong at the $M_{4}$ and $M_{5}$ edges of actinides, because they correspond to dipole allowed transitions to the highly $5 f$ polarized shell. In the case of rare earths, the dipole transitions at the $L_{3}$ edge promote a $2 p$ core electron into the indirectly polarized $d$-bands, while the quadrupolar transitions take it into the magnetized $4 f$-shell. In this case, the two types of transitions have to be taken into account. For $3 d$ metals, the $L$ edges correspond to wavelengths of the order of $17 \AA$ which are too long to allow diffraction experiments. The $K$ edges are the only edges available for scattering experiments. 


\section{Applications of synchrotron X-rays to magnetic scattering}

The applications of synchrotron $\mathrm{X}$-ray scattering to the study of magnetism are threefold. First, the nonresonant scattering cross-section allows experimental determinations of $L$ and $S$ in any type of magnetic ordered compounds. Advantages can be taken from the large flux of photons and the high $Q$ resolution available on synchrotron sources to examine phase transitions [27] and detailed features of magnetic structures [28]. Even magnetic form factors can be obtained at quite large $\boldsymbol{Q}$. The determination of magnetic structures of bulk materials with X-rays appear to be extremely difficult because of the weak scattering cross-section (1), which is the crippling flaw of the nonresonant magnetic X-ray scattering. The second application, the resonant scattering, has alleviated this problem. The large enhancements can be used to study systems with weak magnetic moments [29] or small scattering volume such as micro-crystals or surfaces and interfaces [30]. Furthermore, the resonant scattering is species selective: by tuning the photon energy, it is possible to turn on and off the resonant signal from one element in a magnetic compound; even different valence states can be distinguished [31]. Finally, the line shape analysis of the resonance provides a new spectroscopy technique of excited electronic states. Even the non-magnetic X-ray Thomson scattering can be used to study magnetism through magnetostrictive phenomena. The high $\boldsymbol{Q}$-resolution offered by synchrotron X-ray sources is well suited to the determination of small lattice changes near magnetic phase transitions. Moreover, low and high energy photons can be used to discriminate between "skin" and bulk effects. When solving bulk magnetic structures, neutron diffraction offers most advantages compared to X-rays, simply because neutrons are less absorbed by materials and are less demanding on the sample quality. Neutrons can probe the interior of samples located in sophisticated environments. Nevertheless, we may consider samples with small volume (surfaces, $\mu$-crystals) which cannot be treated as bulk solids and this is where $\mathrm{X}$-rays come into action. Furthermore, the elucidation of particular details in the magnetic arrangements may require special treatments such as high $Q$-resolution scaltering experiments or the separation of different magnetic species, which can be achieved by magnetic X-ray scattering or spectroscopy.

\section{Magnetic X-ray scattering experiments}

Several experiments have been performed within the last few years by using magnetic X-ray scattering. In the following section we select a few experimental examples that illustrate the variety of applications for magnetism in research using synchrotron X-rays. First, nonresonant scattering studies will be presented that deal with the details of magnetic ordering in holmium [28, 32] and magnetic superlattices [33], the separation of $L$ versus $S$ in holmium [7] and the use of white beam diffraction experiments to extract magnetic form factors [34]. Then, resonant magnetic scattering applications will be discussed; we will concentrate on the cases of holmium [35] and UAs [36]. The resonant magnetic X-ray scattering technique can be extended to investigations of magnetic phase transitions, surface ordering, mixed valence materials, etc. 


\subsection{Nonresonant scaltering}

Let us consider metallic holmium. Ho has an hep crystal structure with two layers per chemical unit cell. Below the Néel temperature, holmium exhibits a simple antiferromagnetic spiral structure that manifests itself by the occurrence of symmetric pairs of magnetic satellites around each main Bragg peak along the $c$ axis of the hexagonal structure. The hexagonal basal planes are ferromagnetic, but the moments rotate from plane to plane with an angle of the order of $30^{\circ}-50^{\circ}$ given by the position $\tau$ of the satellites along the $c^{*}$-axis. The positions of these peaks were monitored during high $Q$ resolution $\mathrm{X}$-ray scattering experiments. The $\mathrm{X}$-ray results are in qualitative agreement with ancient neutron data but new features emerged. First, lock-in was observed near commensurate values for $\tau$. Second, extra diffraction peaks were observed, with slightly broader width but comparable intensity when the magnetic peaks are close to a commensurate position. Linear polarization analysis showed that these broader peaks have the same polarization as the incident beam, while, as expected from (2), the magnetic peaks have a rotated component. The extra peaks are due to charge scattering and arise from magneto-elastic couplings. These findings led to a unified model of magnetic structure in rare earths which exhibit modulated magnetic structures. However, in-plane anisotropy favors some crystallographic directions (the six equivalent easy directions in the basal plane) and an incommensurate structure, induced by exchange couplings, costs some energy. To accommodate to the lattice periodicity, some spin discommensurations (spin-slips) appear. Spins along the $c$ axis are normally grouped in pairs that are parallel to the easy directions. A spin-slip corresponds to a single layer associated with an easy direction. Due to magneto-elastic interactions, this defect in the magnetic structure induces a lattice distortion. If the spin structure is periodic, then a corresponding lattice modulation should appear. This is the origin of the extra diffraction peaks mentioned above. This has been generalized to other rare earths [28] and also verified by very careful neutron scattering experiments [37].

During further experiments, we investigated the polarization of the scattered magnetic intensities from lıolmium. The incident X-ray beam was $\sigma$-polarized $(p=0.77)$. The linear degree of polarization of scattered beams was measured for magnetic satellites around different crystal Bragg peaks. The experimental results are displayed in Fig. 1. The solid line was obtained from calculations based on Eq. (2) applied to the structure of holmium. Orbital and spin form factors were taken from electronic orbital calculations. The upper part of the figure shows that the polarization of the scattered beam is progressively rotated with increasing scattering vector due to the presence of the orbital moment. The $L / S$ ratio is shown in the bottom part as a function of scattering vector. Within the experimental errors, the data are consistent with the calculated values represented by the solid line. Further improvements of this techniques are under way at ESRF where higher linear degree of polarization can be achieved. The distinction between orbital and spin magnetization is important to the fundamental understanding of the electronic structure of magnetic materials. Mixed valence materials, heavy fermions and actinides compounds would benefit iom such studies because the orbital and spin densities are not accurately known. 


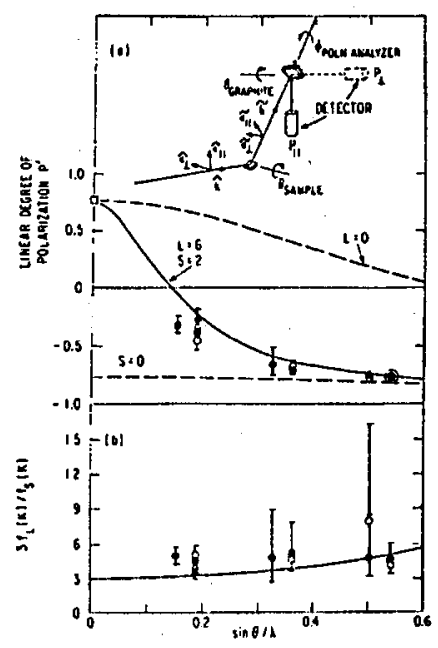

Fig. 1. Polarization analysis of the magnctic X-ray scattering in holmium [7]. Part (a) gives the linear degrec of polarization $P^{\prime}$ of scattered beams from magnetic satellites $(0,0, l \pm \tau)$. The dashed line are calculated assuming $L=0$ and $S=0$. The measured ratio of orbital and spin form factors are sloown on the lower part (b).

In the next example [33], the tunability of the $\mathrm{X}$-ray energy from a synchrotron has been exploited to maximize the cross term between the charge scattering and the magnetic scattcring (1) from ferromagnetic materials. As mentioned above, there always exists an interference term independent of the polarization of the X-ray beam which is similar to the flipping ratio familiar to neutron scatterers. This technique was applied to determine the modulation in the magnetic moment in superlattices composed of alternating gadolinium and non-magnetic yttrium. In the case of a perfect onc-dimensional chemical structure, the reciprocal lattice consists of points located at $Q(l, m)=2 \pi(l / c+m / \Lambda)$ where $c$ is the average lattice constant and $A$ is the modulation wavelength. The two integers $l$ and $m$ denote the average Bragg reflections and their harmonics, respectively. We measured the flipping ratio at several satellite positions as a function of photon encrgy around the $L_{2}$ and $L_{3}$ gadolinium edges (Fig. 2). It is seen from (3) that an accurate determination of the structure factors is required before analyzing the flipping ratio to extract the magnetic structure factors. The use of anomalous $\mathrm{X}$-ray scattering is an excellent tool for such determinations. In order to determine the modulation in the magnetic moment, the flipping ratios for reflection $Q(l, m)$ were determined over several $l$ and $m$ to cover a wide range in intensity (five orders of magnitude) and flipping ratios. The results shown in Fig. 2 for the $\mathrm{Gd}_{21} Y_{21}$ superlattice are consistent with a smootlı reduction in the Gd moment near the interfaces. There is no magnetically dead layers and no alloying was detected. This is the first example of magnetic structure determination with X-rays.

The flipping ratio technique can be extended to white beam experiments [38] in which several Bragg peak signals arc recorded at once. The flipping ratios are 


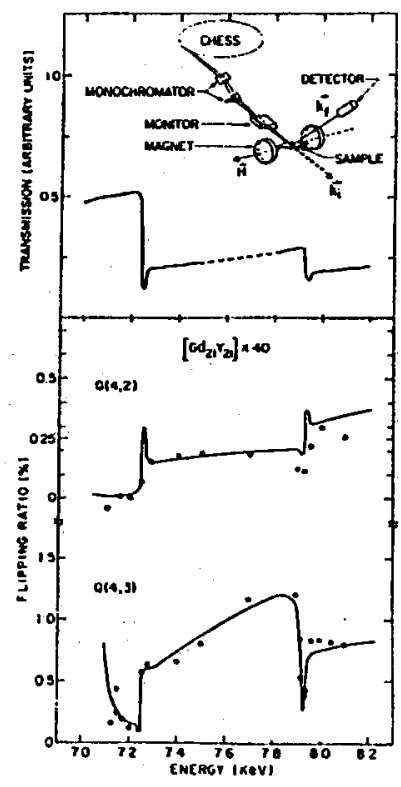

Fig. 2. Flipping ratios as observed (circles) and calculated (solid lines) through the $L_{3}$ and $L_{2}$ absorption edges of gadolinium in a $\mathrm{Gd}_{21} Y_{21}$ multilayer [33]. Note that no resonant magnetic scattering is observed in this geometry.

optimized by choosing a scaltcring angle $20=90^{\circ}$. Bending magnets or asymmetric wigglers on synclirotron offer a certain degree of elliptical polarization out of the orbit plane; furthermore, they can provide photons up to roughly $20 \mathrm{keV}$. Large momentum transfers beyond $10 \AA^{-1}$ can be accessed, much further than neutron scattering data. The experiments have been performed on iron [37] on poorly polarized X-ray beam. The data extend up to $Q=1.3 \AA^{-1}$ and complement neutron results which fall off rapidly with increasing momentum transfer. The data analysis is rather cumbersome, but the method can be improved at shown by recent results obtained at ESRF [39] on Fe which extend up to $Q=25 \AA^{-1}$ at the $(12,0,0)$ Bragg peak.

\subsection{Resonanl scallering}

In the resonant regime, the large enhancement of the magnetic scattering makes feasible the observation of microscopic magnetic phenomena in a wider class of materials than anticipated. We have discussed above the advantages of the resonant techniques. The limitations arise from the Bragg cut-off restriction. In the soft X-ray range below $3 \mathrm{keV}$ ( $L$ edges of $3 d$ metals), only reflectivity or X-ray absorption experiments can be performed. Resonant magnetic X-ray diffraction has been performed on most rarc-carth metals [40-42] and intermetallics [43], on some $d$ compounds $[44,45]$ and a large variety of aclinides $[8,36,46,47]$. We will concentrate on a review of the first experimental evidence for resonant scattering in holmium [35]. Then, we will present the dramatic enliancement discovered at 
the $M$ edges of uranium in UAs [36], followed by the extension of this teclnique to physical problems involving magnetic order and phase transitions near surfaces.

In holmium, a fiftyfold resonant enhancement of the magnetic satellite at position $\tau$ discussed above was discovered as the incident $\mathrm{X}$-ray energy was tuned through the $L_{3}$ absorption edge of holmium $[7,35]$. At that time, counts rates for the magnetic peaks were about $70.000 \mathrm{cts} / \mathrm{s}$ for an incident flux of $10^{11}$ photons/s (recent measurements at ESRF have given count rates of roughly $500,000 \mathrm{cts} / \mathrm{s}$ from the same sample [48]). In addition to the enhancement, resonant harmonics were also observed at positions $2 \tau, 3 \tau$ and $4 \tau$. The first and second harmonics have an asymmetric line shape, whereas the third and fourth order peaks are symmetric but take their maximum at the same energy as the first and second harmonics. The data can be understood on the basis of multipole contributions to the resonant scattering as eluded to above. At the $L_{3}$ edge of holmium, the dipole allowed transitions which couple $2 p$ and spin-down and spin-up $5 d$ orbitals lead to the first and second harmonics. Similarly, the quadrupolar contributions coupling the $2 p$ to $4 f$ orbitals lead to four harmonics at $\tau, 2 \tau, 3 \tau$ and $4 \tau$. The position in energy of the high order peaks corresponds to quadrupole transitions. The asymmetry in the low order satellites is due to the mixing of dipole and quadrupole allowed transitions. From the observed energy line shape of magnetic satellites, it was possible to extract the energy shift between the dipole and quadrupole excitations. The polarization of the scattered intensities was found to follow the predictions of the model presented above. These experiments clearly established the resonant character of the observed intensity: existence of high order satellites and polarization dependence.

Although the resonance at the $L_{3}$ edges of rare earth provides sizable scattered intensities, even larger enliancements were predicted at the $M$ edges. Indeed, resonant scattering amplitudes approaching $9 r_{0}$ have been observed in $U \Lambda s$ [36]. UAs is a simple antiferromagnetic compound with a fcc crystal structure. Below the Néel temperature $T_{\mathrm{N}}=122 \mathrm{~K}$, UAs develops a type $I$ antiferromagnetic order. The phase transition is of first order as observed with neutron scattering [49]. At $T=63 \mathrm{~K}$ another first order transition takes place towards at type-IA phase characterized by propagation vectors $(0,0,1 / 2)$. Using $X$-ray scattering techniques, we monitored the energy dependence of magnetic scattered intensity [36] in Fig. 2: Two huge resonances are evident at 3.55 and $3.73 \mathrm{keV}$, the $M_{5}$ and $M_{4}$ edges of uranium. The nonresonant scattering observed at higher energies is reduced by 7 orders of magnitude. Recent experiments at ESRF have shown that resonant scattering in UAs can be observed at $L_{3}$ edge, but with a much weaker enhancement [50].

With such large intensitics, it is possible to study weak magnetic signals arising from cither small scattering volumes or weak magnetic moments. Small scattering volumes can be found in micro-crystals or "surfaces". Weak moments may also correspond to critical fluctuations. Let us consider two examples of experiments: the formation of antiferromagnetic layers in UAs [30] and the observation of critical length scales in NpAs [47]. 


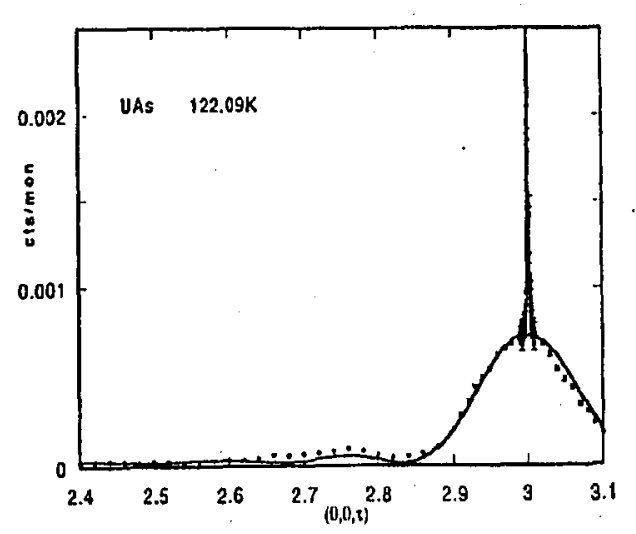

Fig. 3. Resonant magnetic X-ray scattering from UAs [30] ncar the onset of bulk antiferromagnetic order. The narrow and broad components correspond to bulk antiferromagnetism and surface antiferromagnetism.

In UAs, we studied the first-order phase transition with resonant X-ray scattering at the $M_{1}$ edge. A typical scan through the incipient Bragg peak (003) taken at the onset of bulk antiferromagnetism is slown in Fig. 3. The bulk and surface antiferromagnetic responses manifest as the narrow and broad components respectivcly. As a function of temperature, the number of surface antiferromagnetic layers increases on approaching the bulk ordering temperature, while the scattering strength of each layer remains constant. On crossing $T_{N}$, the magnitude of the scattering moment in these layers falls by $20 \%$, while the bulk moment increases dramatically to produce the first-order step. 'This is the first evidence for antiferromagnetic ordering in surface layers. Note that such experiments require a high resolution in momentum space coupled with a high statistical accuracy (the scattered intensities extend over five orders of magnitude).

In $\mathrm{NpAs}$, magnetic critical scatlering was observed by setting the photon energy at the $M_{4}$ edge of neptunium $(3.85 \mathrm{keV})$. Below $T_{N}=173 \mathrm{~K} \mathrm{~Np} A$ s exhibits a continuous transition towards an incommensurate longitudinal antifcrromagnetic structure [51]. The integrated intensity of magnetic Bragg peaks was studied below $T_{\mathrm{N}}$, yielding a critical exponent $\beta=0.36 \pm 0.02$ in agreement with previous neutron data. Above $T_{N}$, scans through the magnetic scattering response revealed that the critical scattering consists of two components: the nornal "broad" component also observed with neutrons, and a "sharp" component corresponding to correlation length 5 or 6 times longer. Similar features have been observed in holmium [52]. The origin of these effects remains unclear up to this moment.

Another application of the resonant $X$-ray scaltering is to distinguish different magnetic elements in the same materials or to study separatcly several valence states that exist on the same ion by tuning the photon energy from one edge to another. In magnetic alloys containing diflerent magnetic atoms, it is possible to monitor the behavior of each constituent by tuning the photon energy through the proper absorption edge and obscrving the corresponding resonant magnetic signal 
[43]. In such a case, the experiments give a direct measurement of the relative resonant strength of each constituent, which can be compared to the extension of some atomic orbitals in the material. This can be applied to different valence states. In particular, studies of the mixed valent material TmSe [31] have demonstrated that the long range magnetic order sets in for the two valence states of $\mathrm{Tm}$ ion in Trme.

\section{Summary}

The field of applications of X-rays to magnetism is much broader than presented in this review. The absorption and spectroscopy techniques play a crucial role in the observation of magnelic properties of surfaces and interfaces. The inelastic scattering methods provide information about the electronic band structures that are responsible for the onset of magnetism. Restricting ourselves to scattering techniques that probe static properties, we have shown that X-ray magnetic Bragg scattering has become a very valuable tool. Even if determination of usual magnetic structures in bulk solids is still the realm of neutron diffraction, the development of X-ray techniques on synchrotron sources and the discovery of resonant X-ray scattering have opened new areas of research in magnetism.

\section{Acknowledgments}

I am pleased to thank F. de Bergevin, N. Bernhoeft, P. Carra, D. Gibbs, G. Grübel, and A. Stunault for many illuminating discussions. I am grateful to the organizers of the ISSRNS'94 for their invitation to present this review. Part of the work by the ESRF group reported here has been supported by a grant from the European Community contract SCI*0467-M(SMA).

\section{References}

[1] M. Faraday, Trans. R. Soc. (London) 5, 592 (1846).

[2] J. Kerr, Philos. Mag. 3, 339 (1877).

[3] G. Schütz, W. Wagner, W. Willelm, P. Kienle, R. Zcller, R. Frahm G. Materlik, Phys. Rev. Lelt. 58, 737 (1987).

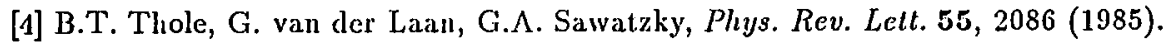

[5] J.D. Jackson, Classical Electrodynamics, Wiley, New York 1975, p. 453.

[6] F. de Bergevin, M. Brunel, Phys. Lett. A 39, 141 (1972).

[7] D. Gibbs, D.R. Harshmann, E.D. Isaacs, D.B. McWhan, D. Mills, C. Vettier, Phys. Rev. Lelt. 61, 1241 (1988).

[8] E.D. Isaacs, D.B. McWhan, C. Pcters, G.E. Ice, D.P. Siddons, J.B. IIastings, C. Vettier, O. Vogt, Phys. Rev. Lelt. 62, 1671 (1989).

[9] F. de Bergevin, M. Bruncl, Acla Crystallogr. A 37, 314 (1981).

[10] M. Gell-Mann, M.L. Golberger, Phys. Rev. 96, 1433 (1954).

[11] P.M. Platzman, N. Tzoar, Phys. Rev. B 2, 3536 (1970).

[12] M. Blume, J. Appl. Phys. 57, 3615 (1985). 
[13] M. Blume, D. Gibbs, Pliys. Rev. B 37, 1779 (1988).

[14] E. Balcar, S.W. Lovesey, in: Theory of Magnetic Neutron and Photon Scattering, Clarendon Press, Oxford 1989.

[15] C. Vettier, D.B. McWhan, Physica B 159, 106 (1989).

[16] W. Schülke, in: Handbook on Synchrotron Radiation, Vol. 3, Eds. G. Brown, D.E. Moncton, Elsevier Scicnce Pub. North-Holland, Amsterdam 1991, p. 565.

[17] M.J. Cooper, S.P. Collins, S.W. Lovescy, D. Laundy, D.N. Timmins, Phys. Scr. Vol. T 35, 103 (1991); R.S. Ilolt, D. Laundy, D.A. Cardwell, M.J. Cooper, T. Naylor, S. Manninen, P. Iatton, Nucl. Instrum. Methods Phys. Res. A 243, 608 (1986).

[18] M.J. Cooper, D. Laundy, D.A. Cardwell, D.N. Timms, R.S. Holt, G. Clark, Phys. Rev. B 34, 5984 (1986).

[19] J.P. Hannon, G.T. Trammell, M. Blume, D. Gibbs, Phys. Rev. Lett. 61, 1241 (1988).

[20] M. Blume, in: Proc. Int. Conf. on Anomalous Scaltering, Malente 1992, North-Holland, Amsterdam 1994.

[21] P. Carra, M. Altarelli, Phys. Rev. Lelt. 64, 1286 (1990).

[22] P. Carra, private communication.

[23] P. Carra, M. Altarelli, F. de Bergevin, Phys. Rev. B 40, 7324 (1989).

[24] P. Carra, B.N. Harmon, B.T. Thole, M. Altarclli, G. Sawatzky, Phys. Rev. Lett. 66, 2495 (1991).

[25] B.T. Thole, P. Carra, F. Sette, G. van der Laan, Phys. Rev. Lelt. 68, 1943 (1992).

[26] P. Carra, B.T. Thole, M. Altarelli, X. Wang, Phys. Rev. Lelt. 70, 694 (1993).

[27] J.P. Hill, T.R. Thurston, R.W. Erwin, M.J. Ramstad, R.J. Birgeneau, Phys. Rev. Lett. G6, 3281 (1991).

[28] J. Bohr, D. Gibbs, J.D. Axe, D.E. Moncton, K.L. d'Amico, C.F. Majkrzak, J. Kwo, M. Hong, C.L. Chien, J. Jensen, Physica $B$ 159, 93 (1989).

[29] E.D. Isaacs, D.B. McWhan, R.N. Kleiman, D.J. Bishop, G.E. Ice, P. Zschak, B.D. Gaulin, T.E. Mason, J.D. Garett, W.L. Buyers, Phys. Rev. Lett. 65, 3185 (1990).

[30] N. Bernhoeft, A. Stunault, C. Vettier, F. de Bergevin, D. Gibbs,T.R. Thurston, S.M. Shapiro, J.B. Ilastings, P. Dalmas, G. Helgesen, O. Vogt, Int. Conf. on Magnetism, Warsaw, August 1994, to be published.

[31] D.B. McWhan, E.D. Isaacs, P. Carra, S.M. Shapiro, B.T. Thole, S. Hoshino, Phys. Rev. B 47, 8630 (1993).

[32] D. Gibbs, D.E. Moncton, K.L. d'Amico, J. Bohr, B.II. Grier, Phys. Rev. Lett. 55, 234 (1985); D. Gibbs, J. Bohr, J.D. Axe, D.E. Moncton, K.L. d'Amico, Phys. Rev. $B$ 34, 8182 (1986).

[33] C. Vettier, D.B. McWhan, E.M. Gyorgy, J. Kwo, B.M. Buntschuh, B.W. Batterman, Phys. Rev. Lelt. 56, 757 (1986).

[34] S.P. Collins, D. Laundy, A.J. Rollason, Philos. Mag. 65, 37 (1992).

[35] D. Gibbs, G. Grübel, D.R. Harshman, E.D. Isaacs, D.B. McWhan, D. Mills, C. Vettier, Phys. Rev. B 43, 5663 (1991).

[36] D.B. McWhan, C. Vettier, E.D. Isaacs, G.E. Ice, D.P. Siddons, J.B. Hastings, C. Peters, O. Vogt, Phys. Rev. B 42, 6007 (1990).

[37] R.A. Cowley, S.B. Bates, J. Phys. C 21, 4113 (1988); R.A. Cowley, D.A. Jehan, D.F. McMorrow, G.J. McIntyre, Phys. Rev. Lelt. 66, 1521 (1991). 
[38] D. Laundy, S.P. Collins, A.J. Rollason, J. Phys., Condens. Matter 3, 369 (1991).

[39] R.D. Bateson, P. Suortti, A.K. Freund, to be published.

[10] E.D. Isaacs, D.B. McWhan, D.P. Siddons, J.B. Hastings, D. Gibbs, Phys. Rev. B 40, 9336 (1989).

[41] J. Bohr, D. Gibbs, K.G. Huang, Phys. Rev. B 42, 4322 (1990).

[42] M.K. Sanyal, D. Gibbs, J. Bohr, M. Wulff, Phys. Rev. B 49, 1079 (1994).

[43] A. Stunault, C. Vettier, F. de Bergevin, F. Maier, G. Grübel, R.M. Galera, S.B. Palmer, Int. Conf. Magnetism, Warsaw, August 1994, to be published.

[44] K. Namikawa, M. Ando, T. Nakajima, H. Kawata, J. Phys. Soc. Jpn. 54, 4099 (1985).

[45] F. de Bergevin, M. Brunel, R.M. Galera, C. Vettier, E. Elkaïm, M. Bessière, S. Lefèbvre, Phys. Rev. B 42, 10772 (1992).

[16] C.C. Tang, W.G. Stirling, G.II. Lander, D. Gibbs, W. Ilerzog, P. Carra, K. Mattemberger, O. Vogt, Pliys. Rev. B 46, 5287 (1992).

[47] S. Langridge, W.G. Stirling, G.H. Lander, J. Rebizant, J.C. Spirlet, D. Gibbs, O. Vogt, Europhys. Lelt. 25, 137 (1994).

[18] G. Grübel, C. Vettier, D. Gibbs, J. Bohr, to be published.

[49] S.K. Sinha, G.II. Lander, S.M. Shapiro, O. Vogt, Phys. Rev. B 23, 4556 (1981).

[50] N. Bernhoeft, A. Stunault, C. Vettier, F. de Bergevin, G. Grübel, to be published.

[51] P. Burlet, D. Bonnisscau, J. Rossat-Mignod, J.C. Spirlet, J. Rebizant, O. Vogt, J. Magn. Magn. Maler. 63-64, 151 (1989).

[52] T.R. Thurtson, G. Helgesen, D. Gibbs, J.P. Hill, B.D. Gaulin, G. Shirane, Phys. Rev. Lelt. 70, 3281 (1993). 